\title{
Clinical and Etiological Characteristics of Atypical Hand-Foot-and-Mouth Disease in Children from Chongqing, China: A Retrospective Study
}

\author{
Xiang Yan, ${ }^{1,2}$ Zhen-Zhen Zhang,' Zhen-Hua Yang, \\ Chao-Min Zhu, ${ }^{1}$ Yun-Ge Hu, ${ }^{1}$ and Quan-Bo Liu ${ }^{1}$ \\ ${ }^{1}$ Department of Infectious Disease, Children's Hospital of Chongqing Medical University, Ministry of Education Key Laboratory of \\ Child Development and Disorders, Chongqing Key Laboratory of Pediatrics, Chongqing 400014, China \\ ${ }^{2}$ Department of Epidemiology, School of Public Health, University of Michigan, M5124 SPH II, 1415 Washington Heights, \\ Ann Arbor, MI 48109, USA
}

Correspondence should be addressed to Quan-Bo Liu; liuquanbo_lqb@163.com

Received 29 April 2015; Revised 29 August 2015; Accepted 7 September 2015

Academic Editor: Niwat Maneekarn

Copyright ( 92015 Xiang Yan et al. This is an open access article distributed under the Creative Commons Attribution License, which permits unrestricted use, distribution, and reproduction in any medium, provided the original work is properly cited.

\begin{abstract}
Background. Hand-foot-and-mouth disease (HFMD) is a disease that had similar manifestations to chickenpox, impetigo, and measles, which is easy to misdiagnose and subsequently causes delayed therapy and subsequent epidemic. To date, no study has been conducted to report the clinical and epidemiological characteristics of atypical HFMD. Methods. 64 children with atypical HFMD out of 887 HFMD children were recruited, stool was collected, and viral VP1 was detected. Results. The atypical HFMD accounted for $7.2 \%$ of total HFMD in the same period (64/887) and there were two peaks in its prevalence in nonepidemic seasons. Ten children (15.6\%) had manifestations of neurologic involvement, of whom 4 (6.3\%) were diagnosed with severe HFMD and 1 with critically severe HFMD, but all recovered smoothly. Onychomadesis and desquamation were found in 14 patients (21.9\%) and 15 patients (23.4\%), respectively. The most common pathogen was coxsackievirus A6 (CV-A6) which accounted for 67.2\%, followed by nontypable enterovirus (26.6\%), enterovirus 71 (EV-A71) (4.7\%), and coxsackievirus A16 (A16) (1.5\%). Conclusions. Atypical HFMD has seasonal prevalence. The manifestations of neurologic involvement in atypical HFMD are mild and usually have a good prognosis. CV-A6 is a major pathogen causing atypical HFMD, but not a major pathogen in Chongqing, China.
\end{abstract}

\section{Introduction}

Hand-foot-and-mouth disease (HFMD) is an infectious disease which mainly affects children under 5 years of age. HFMD is characterized by low-grade fever, general malaise, and vesicles or papulae on the palms, soles, and buttocks.

Since firstly reported in 1957 [1], HFMD began to spread all over the world gradually, which brought heavy economic and social burden to the world. Especially in China, there are several millions of HFMD being reported [2]. Researches showed that enteroviruses were the main pathogens of HFMD, most of which were Enterovirus-A71 (EV-A71) and coxsackievirus A16 (CV-A16) [3, 4]. However, rates of HFMD caused by coxsackievirus A6 (CV-A6) and coxsackievirus A10 have increased by years nowadays in Shenzhen [5], Shandong
[6], Guangdong [7], and so on. Besides, there were also other reported pathogens like coxsackievirus A4-5 as well as coxsackievirus B2-5 [8].

In 2008, HFMD characterized by onychomadesis in later phase was first reported in Finland [9], and CV-A6 was the major pathogen. Thereafter, HFMD caused by CV-A6 has been reported in Spain [10], Japan [11], Thailand [12], USA [13], and Singapore [14]. The incidence of CV-A6 related infections was increasing in above countries, and CV-A6 became a major pathogen. Since 2001, CV-A6 has been reported as one of the top 5 pathogens causing HFMD in Taiwan [15], and the incidence of CV-A6-related HFMD increased every year. In addition, in Guangdong China [16], HFMD caused by CV-A6 also has an increased incidence year after year and CV-A6 has been an important pathogen of 
HFMD. Even an outbreak of HFMD caused by CV-A6 was reported in Changchun, China [17], in 2013. Several studies reported that HFMD caused by CV-A6 was different from previously reported HFMD and characterized by vesicles or papular on the faces, trunks, and limbs or large vesicles or bullae (diameter $>5 \mathrm{~mm}$ ) on any site of the body, as well as onychomadesis and desquamation in later phase. HFMD with above manifestations is also known as atypical HFMD [18].

We have also encountered atypical HFMD in Chongqing, China, in 2013. This disease had similar manifestations to chickenpox, impetigo, and measles. Thus, it is easy to be misdiagnosed, subsequently causing delayed therapy and subsequent epidemic. To date, no study has been conducted to report the clinical and epidemiological characteristics of atypical HFMD. Chongqing is a southwestern city of China with a large population, a relatively poor sanitary condition, and a high incidence of infectious diseases. Thus, we should pay more attention to the prevention of HFMD. This study aimed to summarize the epidemiological, clinical, and etiological characteristics of atypical HFMD diagnosed between September 2013 and August 2014 in Chongqing, China, which may be helpful for the diagnosis, differential diagnosis, and therapy of HFMD.

\section{Material and Methods}

2.1. Patients. A total of 887 hospitalized children were diagnosed with HFMD in the Children's Hospital of Chongqing University between September 2013 and August 2014. Of these patients, 64 children $(7.2 \%, 64 / 887)$ with atypical HFMD were recruited into this study for further analysis. Informed consent was obtained. This study has been approved by the Ethics Committee related to the Children's Hospital of Chongqing University.

2.2. Inclusion Criteria. HFMD patients with following characteristics were recruited into present study. Characteristics of atypical rashes were as follows: (1) distribution: papules and/or vesicles (diameter $<5 \mathrm{~mm}$ ) being also found in other sites such as face, neck, perioral area, trunk, limbs, and externalia besides the palms, soles, and buttocks; and/or (2) morphology of rashes: large vesicles or bullae (diameter $>5 \mathrm{~mm}$ ), erosive lesions, purpuric/petechial lesions, or "eczema coxsackium" at any site $[18,19]$. Detection of enterovirus was positive or serological detection was positive. The clinical diagnosis, etiological diagnosis, and diagnosis of common HFMD and severe HFMD were performed according to the Guideline for the Clinical Diagnosis and Therapy of Hand-Foot-Mouth Disease developed by Chinese Ministry of Health in 2010. Fever was defined as body temperature of $\geq 37.5^{\circ} \mathrm{C}$; onychomadesis was defined when a nail separated from the nail bed (detach) due to the stop of transverse ridge growth or transverse ridge mutilation after long term restriction of parent material activity.

2.3. Exclusion Criteria. (1) Informed consent was not obtained from their parents or their parents withdraw from this study before the end of study; (2) stools were not collected, or the clinical information was incomplete; (3) there were skin lesions caused by other diseases (such as chickenpox, impetigo, and measles).

2.4. Sample Collection. This study was approved by the Ethics Committee of Children's Hospital of Chongqing University (number: 105/2014). Informed consent was obtained from parents of participants before study. Stools were collected from 64 children with atypical HFMD in acute phase (within 3 days after disease onset) and stored at $-80^{\circ} \mathrm{C}$ for further detection. In addition, the rashes were photographed, and onychomadesis, desquamation, and sequelae were closely monitored during follow-up period. The patients' characteristics and clinical manifestations were collected from the medical record. At the same time, the characteristics of all the patients with different types of HFMD were also recorded in the same period for the epidemiological comparisons.

2.5. Virus Detection and DNA Sequencing. RNA was extracted from stool samples by using QIAampH MinElute Virus Spin Kits (Qiagen, Hilden, Germany) and cDNA was synthesized by using Reverse Transcription System A3500 (Promega, Madison, WI, USA) according to the manufacturer's instructions. Nested-PCR was performed to detect universal enteroviruses (EV-U), EV-A71, CV-A16, respectively, while ordinary PCR was performed to detect CV-A6. Primers for CV-A6 were $5^{\prime}$-TGGTAGGAGTTGTGGAGGT-3' (forward) and $5^{\prime}$ CCTTCATAATCYGTAGTGGTT-3' (reverse), which was constructed by ourselves. And primers for other genes were obtained from the study of Ge et al. [20]. Sequencing was performed in 43 samples positive for CV-A6, and results were compared with those in BLAST (www.ncbi.nlm.nih.gov/ blast). Neighbor-joining (NJ) method was employed to construct phylogenetic tree with bootstrap method 1000 as a parameter.

2.6. Statistical Analysis. Data with normal distribution were expressed as mean \pm standard deviation $(\bar{x} \pm s)$. Data with abnormal distribution were presented as range (M). Statistical analysis was performed with SPSS version 17.0 (SPSS Inc., Chicago, IL, USA). $P<0.05$ was considered statistically significant.

\section{Results}

3.1. Incidence of Atypical HFMD in Chongqing. During this study, a total of 887 hospitalized children were diagnosed with HFMD, of whom 7.2\% (64/887) were atypical HFMD. Both total and atypical HFMD had two peaks in their incidences. The proportion of atypical HFMD varied over time and ranged from $1.9 \%$ to $30.4 \%$. The proportion was the highest from January to March, a season transiting from winter to spring, in which atypical HFMD accounted for $30.4 \%(7 / 23)$, $17.6 \%(3 / 17)$, and $12.5 \%(4 / 32)$, respectively. From January to March, the highest proportion of atypical HFMD was found in January. Another peak was found from August to 


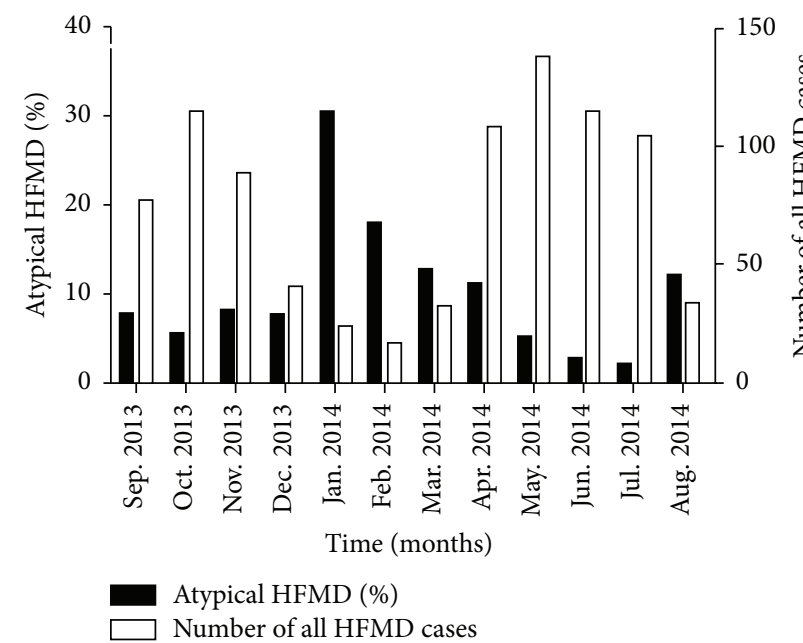

Figure 1: Proportion of atypical HFMD to total HFMD per month and number of total HFMD in each month. Black: proportion of atypical HFMD; white: number of total HFMD. $X$-axis: time; left $Y$-axis: proportion of atypical HFMD; right $Y$-axis: number of total HFMD.

October in which the proportion of atypical HFMD was $12.1 \%$ (4/33), 7.8\% (6/77), and 5.3\% (6/114), respectively. Besides, atypical HFMD was also found in other seasons. However, total HFMD peaked from April to July, a season transiting from spring to summer, in which total HFMD accounted for about $52.2 \%$ in the study period (443/887). The second peak was found from September to November, a season transiting from autumn to winter, and total HFMD in these months accounted for $31.5 \%$ in the study period (279/887) (Figure 1).

3.2. Demographic Characteristics of Atypical HFMD. Among the 64 children with atypical HFMD, there were 40 males and 24 females with the male to female ratio of $1.67: 1$. The age of children ranged from 6 months to 48 months (median: 15 months). Atypical HFMD mainly occurred in children younger than 3 years, which accounted for 93.8\% (60/64), and $54.7 \%(35 / 64)$ of children were younger than 1 year. Most patients lived in the city $(62.5 \%, 40 / 64)$. A majority of children lived scattered $(79.7 \%, 51 / 64)$ and remaining children were on nursery care $(15.6 \%, 10 / 64)$ and schooling $(4.7 \%, 3 / 64)$ (Table 1).

3.3. Clinical Manifestations. Atypical HFMD children usually presented with fever $(79.7 \%, 51 / 64)$, poor appetite $(67.2 \%$, $43 / 64)$, and salivation $(64.1 \%, 41 / 64)$. In addition, 10 children had manifestations of neurologic involvement of whom the startle response $(15.6 \%, 10 / 64)$, vomiting $(7.8 \%, 5 / 64)$, and convulsion $(12.5 \%, 8 / 64)$ had higher prevalences, but headache and limb trembling were found in only 2 patients, unconsciousness in 1 and unsteady gait in 1 . Severe atypical HFMD was observed in 5 patients $(7.8 \%, 5 / 64)$, of whom 4 had severe atypical $\operatorname{HFMD}(6.3 \%, 4 / 64)$ and 1 had critically severe atypical $\operatorname{HFMD}(1.5 \%, 1 / 64)$, but all these children recovered smoothly after therapy without any sequela (Table 1).
TABLE 1: Demographic and clinical presentations of patients with atypical HFMD.

\begin{tabular}{lcc}
\hline Variables & $\begin{array}{c}\text { Atypical HFMD }(n=64) \\
n(\%)\end{array}$ & $95 \%$ CI \\
\hline $\begin{array}{l}\text { Demographics } \\
\text { Age (years) }\end{array}$ & & \\
$\leq 1$ & $35(54.7)$ & $53.7 \% \sim 55.7 \%$ \\
$1-3$ & $25(39.1)$ & $37.9 \% \sim 40.3 \%$ \\
$>3$ & $4(6.2)$ & $5.5 \% \sim 6.9 \%$ \\
Sex & & \\
$\quad$ Male & $40(62.5)$ & $61.6 \% \sim 63.4 \%$ \\
$\quad$ Female & $24(37.5)$ & $36.3 \% \sim 38.7 \%$ \\
Residence & & \\
$\quad$ Urban & $40(62.5)$ & $61.6 \% \sim 63.4 \%$ \\
$\quad$ Rural & $24(37.5)$ & $36.3 \% \sim 38.7 \%$ \\
Children care & & \\
$\quad$ Home care & $51(79.7)$ & $79.1 \% \sim 80.3 \%$ \\
Nursery care & $10(15.6)$ & $14.6 \% \sim 16.6 \%$ \\
Schooling & $3(4.7)$ & $4.1 \% \sim 5.3 \%$
\end{tabular}

Symptoms

Fever

$\geq 37.5^{\circ} \mathrm{C}$

$51(79.7)$

$79.1 \% \sim 80.3 \%$

$<37.5^{\circ} \mathrm{C}$

$13(20.3)$

$19.2 \% \sim 21.4 \%$

Cough

Yes

20 (31.3)

$30.1 \% \sim 32.5 \%$

No

44 (68.7)

67.9\% 69.5\%

Rhinorrhea

Yes

15 (23.4)

$22.3 \% \sim 24.5 \%$

No

49 (76.6)

$76.0 \% \sim 77.2 \%$

Salivation

Yes

41 (64.1)

$63.2 \% \sim 65.0 \%$

No

23 (35.9)

$34.7 \% \sim 37.1 \%$

Diarrhea

Yes

9 (14.1)

$13.1 \% \sim 15.1 \%$

No

55 (85.9)

$85.5 \% \sim 86.3 \%$

Poor oral intake

Yes

43 (67.2)

No

$21(32.8)$

$66.4 \% \sim 68.0 \%$

$31.6 \% \sim 34.0 \%$

Vomiting

Yes

$5(7.8)$

$7.0 \% \sim 8.6 \%$

No

$59(92.2)$

$92.0 \% \sim 92.4 \%$

Headache

Yes

$2(3.1)$

$25.8 \% \sim 36.2 \%$

No

$62(96.9)$

$96.8 \% \sim 97.0 \%$

Febrile seizure

Yes

8 (12.5)

$11.6 \% \sim 13.4 \%$

No

56 (87.5)

$87.1 \% \sim 87.9 \%$

Startle response

Yes

10 (15.6)

$14.6 \% \sim 16.6 \%$

No

$54(84.4)$

$84.0 \% \sim 84.8 \%$

Altered consciousness

\begin{tabular}{lcc} 
Yes & $1(1.6)$ & $1.2 \% \sim 2.0 \%$ \\
No & $63(98.4)$ & $98.3 \% \sim 98.4 \%$ \\
\hline
\end{tabular}


TABLE 1: Continued.

\begin{tabular}{lcc}
\hline Variables & $\begin{array}{c}\text { Atypical HFMD }(n=64) \\
n(\%)\end{array}$ & $95 \%$ CI \\
\hline Limb trembling & & \\
$\quad$ Yes & $2(3.1)$ & $2.6 \% \sim 3.6 \%$ \\
No & $62(96.9)$ & $96.8 \% \sim 97.0 \%$ \\
Unsteady gait & & \\
Yes & $1(1.6)$ & $1.2 \% \sim 2.0 \%$ \\
No & $63(98.4)$ & $98.3 \% \sim 98.4 \%$ \\
\hline
\end{tabular}

HFMD, hand-foot-and-mouth disease. Statistical analysis was performed with SPSS version 17.0 (SPSS Inc., Chicago, IL, USA). A value of $P<0.05$ was considered statistically significant.

3.4. Characteristics of Rashes. Rashes of atypical HFMD were distributed not only in typical sites but on other sites (such as lower limbs [36/64, 56.3\%], face [34/64, 53.1\%], trunk [27/64, 42.2\%], upper limb [19/64, 29.7\%], and externalia $[9 / 64,14.1 \%])$. Rashes on lower limbs were mainly found in the thigh $(17 / 36,47.2 \%)$ and those on face were mainly noted in perioral area $(20 / 34,58.8 \%)$. In respect of the number of sites with involvement, $5-7(59.4 \%, 38 / 64)$ sites were the most common, followed by $2-4(31.3 \%, 20 / 64)$ and $8-9(9.3 \%$, $6 / 64$ ). The maximum number of sites with involvement was 9 . In respect of rash morphology, papula-dominant rashes were found in 44 patients $(68.8 \%, 44 / 64)$ and vesicle-dominant rashes in 41 children $(64.1 \%, 41 / 64), 22$ children had both papula and vesicle $(34.4 \%, 22 / 64), 13$ patients $(20.3 \%, 13 / 64)$ showed large vesicles, 2 had erosive lesions $(3.1 \%, 2 / 64)$ with itching, and $3(4.7 \%, 3 / 64)$ had scabs in late phase (Figure 2 and Table 2).

3.5. Complications in Late Phase: Onychomadesis and Desquamation. All the children received follow-up after therapy. Of these patients, $14(21.9 \%, 14 / 64)$ developed onychomadesis and $15(23.4 \%, 15 / 64)$ had desquamation. In 14 children with onychomadesis, the most common virus in children with onychomadesis was CV-A6 (71.4\%, 10/14), followed by nontypable enterovirus $(14.3 \%, 2 / 14)$ and EV-A71 $(14.3 \%, 2 / 14)$. The mean time to onychomadesis was 4.7 weeks after the acute phase of HFMD (range: 2-7 weeks), and onychomadesis was usually found at 5 th week $(5 / 14,35.7 \%)$. Onychomadesis was mainly found in the right thumb $(8 / 14,57.1 \%)$ and with a mean of 4.7 fingers/toes (range: 1-20). The most common virus in children with desquamation was CV-A6 (80\%; 12/15), followed by nontypable enterovirus $(13.3 \%, 2 / 15)$ and EVA71 $(6.7 \% ; 1 / 15)$. The mean time to desquamation was 4.3 weeks after the acute phase of HFMD (range: 2-8 weeks) and desquamation was usually found at 4 th week $(5 / 15,33.3 \%)$. Of note, desquamation was often found at the sites with rashes $(12 / 15,80.0 \%)$ (Table 2 and Figure 3$).$

3.6. Etiological Characteristics of Atypical HFMD and DNA Sequencing. Among these 64 patients with atypical HFMD, RT-nested PCR showed all the samples were positive for EV-U, and the most common pathogen was CV-A6 (67.2\%; 43/64), followed by nontypable enterovirus, EV-A71, and CVA16 which were found in 26.6\% (17/64), 4.7\% (3/64), and
TABLE 2: Rashes and complications of patients with atypical HFMD.

\begin{tabular}{|c|c|c|}
\hline Variables & $\begin{array}{c}\text { Atypical HFMD }(n=64) \\
n(\%)\end{array}$ & $95 \% \mathrm{CI}$ \\
\hline \multicolumn{3}{|c|}{ Rash distribution } \\
\hline \multicolumn{3}{|c|}{ Palm/soles } \\
\hline Yes & $51(79.7)$ & $79.1 \% \sim 80.3 \%$ \\
\hline No & $13(20.3)$ & $19.2 \% \sim 21.4 \%$ \\
\hline \multicolumn{3}{|l|}{ Oral ulcer } \\
\hline Yes & $47(73.4)$ & $72.7 \% \sim 74.1 \%$ \\
\hline No & $17(26.6)$ & $25.4 \% \sim 27.8 \%$ \\
\hline \multicolumn{3}{|l|}{ Face } \\
\hline Yes & $34(53.1)$ & $52.1 \% \sim 54.1 \%$ \\
\hline No & $30(46.9)$ & $45.8 \% \sim 48.0 \%$ \\
\hline \multicolumn{3}{|l|}{ Torso } \\
\hline Yes & $27(42.2)$ & $41.1 \% \sim 43.3 \%$ \\
\hline No & $37(57.8)$ & $56.8 \% \sim 58.8 \%$ \\
\hline \multicolumn{3}{|l|}{ Arms } \\
\hline Yes & $19(29.7)$ & $28.5 \% \sim 30.9 \%$ \\
\hline No & $45(70.3)$ & $69.5 \% \sim 71.1 \%$ \\
\hline \multicolumn{3}{|l|}{ Legs } \\
\hline Yes & $36(56.3)$ & $55.3 \% \sim 57.3 \%$ \\
\hline No & $28(43.7)$ & $42.6 \% \sim 44.8 \%$ \\
\hline \multicolumn{3}{|l|}{ Buttocks } \\
\hline Yes & $50(78.1)$ & $77.5 \% \sim 78.7 \%$ \\
\hline No & $14(21.9)$ & $20.8 \% \sim 23.0 \%$ \\
\hline \multicolumn{3}{|l|}{ Externalia } \\
\hline Yes & $9(14.1)$ & $13.1 \% \sim 15.1 \%$ \\
\hline No & $55(85.9)$ & $85.5 \% \sim 86.3 \%$ \\
\hline \multicolumn{3}{|c|}{ Morphology } \\
\hline \multicolumn{3}{|l|}{ Vesicle } \\
\hline Yes & $41(64.1)$ & $63.2 \% \sim 65.0 \%$ \\
\hline No & $23(35.9)$ & $34.7 \% \sim 37.1 \%$ \\
\hline \multicolumn{3}{|l|}{ Papula } \\
\hline Yes & $44(68.8)$ & $68.0 \% \sim 69.6 \%$ \\
\hline No & $20(31.2)$ & $30.0 \% \sim 32.4 \%$ \\
\hline \multicolumn{3}{|l|}{ Bulla } \\
\hline Yes & $13(20.3)$ & $19.2 \% \sim 21.4 \%$ \\
\hline No & $51(79.7)$ & $79.1 \% \sim 80.3 \%$ \\
\hline \multicolumn{3}{|l|}{ Erosions } \\
\hline Yes & $2(3.1)$ & $2.6 \% \sim 3.6 \%$ \\
\hline No & $62(96.9)$ & $96.8 \% \sim 97.0 \%$ \\
\hline \multicolumn{3}{|c|}{ Long-term complications } \\
\hline \multicolumn{3}{|c|}{ Desquamation } \\
\hline Yes & $15(23.4)$ & $22.6 \% \sim 24.5 \%$ \\
\hline No & $49(76.6)$ & $76.0 \% \sim 77.2 \%$ \\
\hline \multicolumn{3}{|c|}{ Onychomadesis } \\
\hline Yes & $14(21.9)$ & $20.8 \% \sim 23.0 \%$ \\
\hline No & $50(78.1)$ & $77.5 \% \sim 78.7 \%$ \\
\hline
\end{tabular}

HFMD, hand-foot-and-mouth disease. Statistical analysis was performed with SPSS version 17.0 (SPSS Inc., Chicago, IL, USA). A value of $P<0.05$ was considered statistically significant.

$1.5 \%(1 / 64)$ of patients, respectively. Furthermore, sequencing was performed in 43 samples positive for CV-A6 and the fragment was $630 \mathrm{bp}$ in length. After homology analysis of nucleotides and amino acids, results showed the homology of 


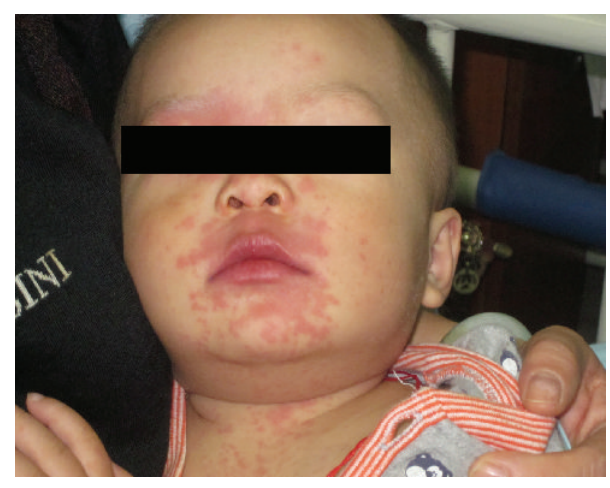

(a)

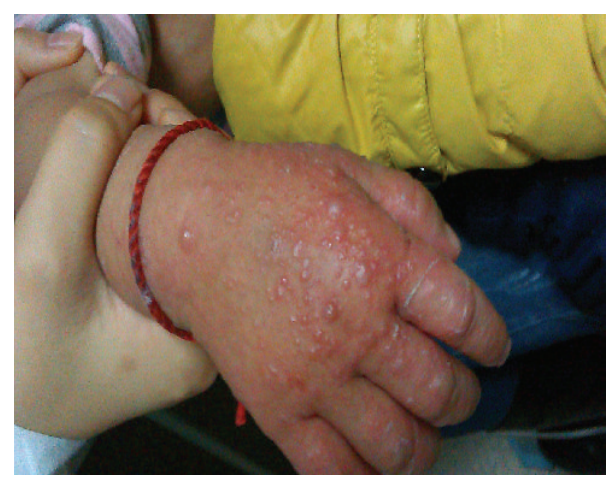

(c)

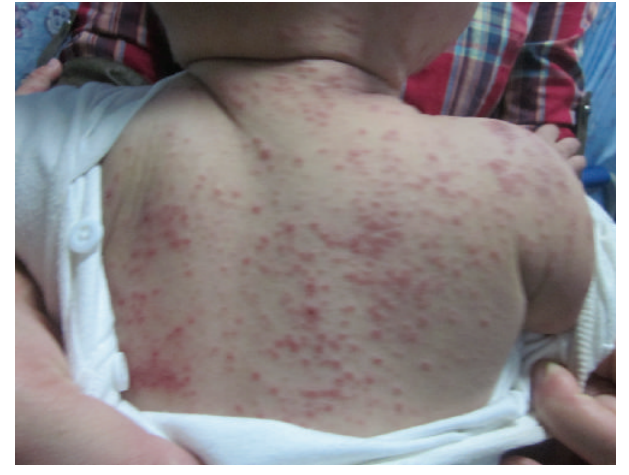

(b)

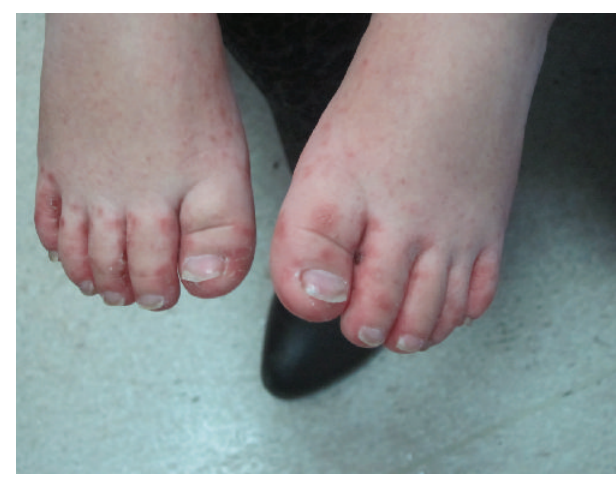

(d)

FIGURE 2: Characteristics of rashes in atypical HFMD. (a) A boy aged 1 year, and papulae were mainly distributed on the perioral area and face; (b) a boy aged 1 year and 1 month, and papulae/vesicles were found at the back; (c) a girl aged 1 year and 6 months, and papulae were found on the hand; (d) a boy aged 1 year and 8 months, and erosions were noted in both feet.

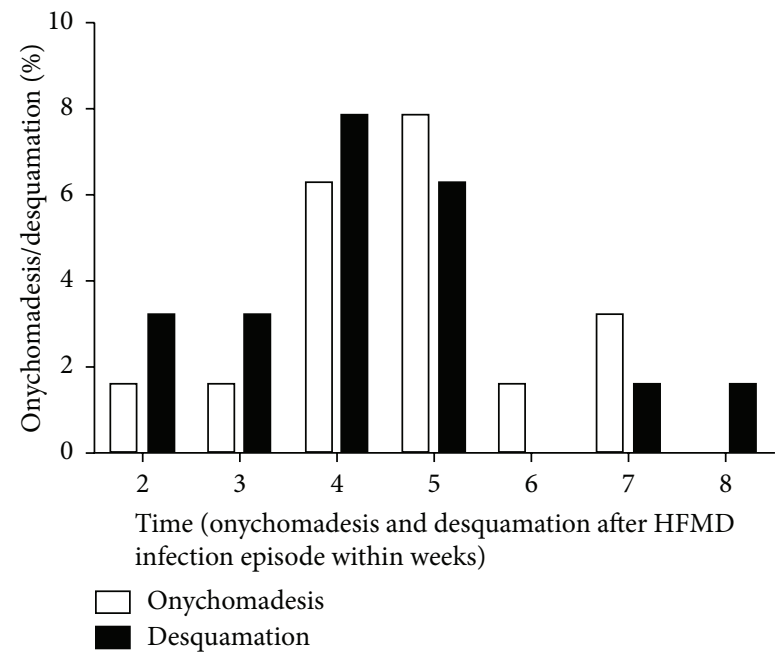

FIGURE 3: Incidences of onychomadesis and desquamation in atypical HFMD children at different time points. $X$-axis: time of follow-up after acute phase; $Y$-axis: proportion of children with onychomadesis/desquamation in children receiving follow-up. Statistical analysis was performed with SPSS version 17.0 (SPSS Inc., Chicago, IL, USA). A value of $P<0.05$ was considered statistically significant.
CV-A6 was $87.6 \%-100 \%$ and $94.5 \%-100 \%$ in the nucleotides and amino acids, respectively, as compared with the strain found in Spain, Finland, Japan, and Taiwan (Figure 4).

\section{Discussion}

The clinical characteristics of atypical HFMD are usually atypical, which brings difficulty to the clinical diagnosis and therapy of HFMD, which may cause misdiagnosis and delayed therapy, resulting in regional or large-scale epidemic. To date, no study has been conducted to investigate the epidemiological and clinical characteristics of atypical HFMD. In the present study, we for the first time described the epidemiological, clinical, and etiological characteristics of atypical HFMD in Chongqing, China, between September 2013 and August 2014. Our findings may provide significant evidence for the clinical diagnosis, timely isolation, and therapy of atypical HFMD.

Results of this study showed that atypical HFMD had obviously seasonal prevalence, was often found in young children and in urban children, and usually predicted a good prognosis. In atypical HFMD children, rashes were widely distributed, skin lesions were severe, and the incidences of 


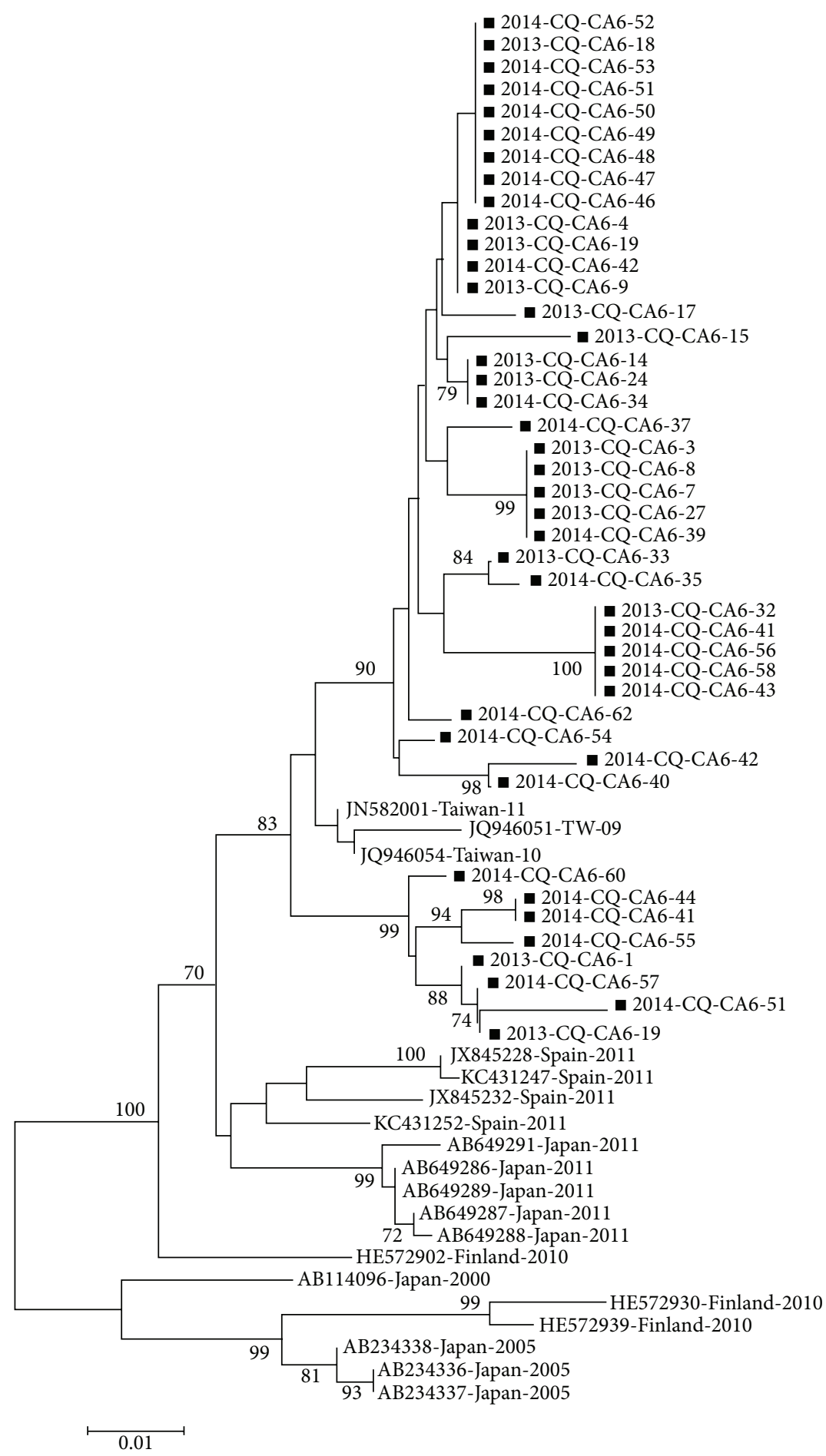

Figure 4: Phylogenetic analysis of coxsackievirus A6 strains based on the partial VP1 gene sequence (nucleotide position: 254-864). $\mathbf{m}$ virus separated in the present study, and remaining viruses were from Genbank including 2011 Spain strains (JX845228, JX845232, KC431247, and KC431252), 2000-2011 Japan strains (AB114096, AB234336, AB234337, AB234338, AB649286, AB649287, AB649288, AB649289, and AB649291), 2010 Finland strains (HE572902, HE572930, and HE572939), and 2009-2011 Taiwan strains (JN582001, JQ946051, and JQ946054).

onychomadesis and desquamation were relatively high in late phase of atypical HFMD. Further etiological analysis showed CV-A6 was a major pathogen causing atypical HFMD, which had a high homology to the strain found in Finland, Taiwan, and other regions.
In the study period, the prevalence of atypical HFMD showed seasonal characteristics, and its incidence varied in different seasons. The incidence of atypical HFMD was high in the seasons transiting from winter to spring and from summer to autumn, but that of total HFMD was high in the 
seasons transiting from spring to summer and from autumn to winter. That is, the peak of atypical HFMD occurred in the troughs of total HFMD, but that of total HFMD occurred in the troughs of atypical HFMD. It is well known that the pathogenesis of HFMD is closely related to climatic factors such as temperature, humidity, and rainfall. The higher the humidity and temperature, the higher the possibility the presence of HFMD has within a certain range [21]. Previous studies [22, 23] have shown that HFMD peaks in the season transiting from spring to summer, followed by the season transiting from autumn to winter, which is consistent with our findings. In Chongqing, China, the peaks of atypical HFMD occurred alternatively with the total HFMD. This was not consistent with the findings that the peak of total HFMD overlaps that of atypical HFMD in Taiwan and Japan [18, 24]. However, whether the characteristics of atypical HFMD were related to the geographical region, climate, race, and survival conditions of CV-A6 (optimal temperature, humidity, and wind velocity for CV-A6 growth) and whether there was lack of temperature sensitive amino acids [25] are still unclear, and the specific mechanisms are required to conduct further investigations. This also reminds us that it is necessary to prevent HFMD in the HFMD prevalent seasons and also pay attention to the seasons of atypical HFMD to prevent regional or large scale epidemic.

In our study, atypical HFMD was mainly found in children younger than 3 years old, and more than $50 \%$ children (54.7\%) were younger than 1 year old. This was similar to findings in the study of Puenpa et al. [12]. Whether the young age is associated with special features of CV-A6 or mutation of CV-A6 in atypical HFMD is still poorly understood, but these findings indicate the correlation between atypical HFMD and young age. This reminds us that we should emphasize the prevention and monitoring of atypical HFMD in children younger than 1 year.

In the present study, only $15.6 \%$ of children with atypical HFMD had manifestations of neurologic involvement. Further analysis showed 4 cases were severe HFMD and 1 was critically severe HFMD, but all of these children recovered smoothly after therapy without sequelae, suggesting a good prognosis. This was in agreement with findings reported by Huang et al. [18]. This indicates that atypical HFMD has a good prognosis but still has a possibility to cause severe HFMD, or even critically severe HFMD, and clinicians should pay attention to atypical HFMD. Few studies have been conducted to report the clinical characteristics of atypical HFMD and more studies are required to investigate the prognosis of atypical HFMD.

In children with atypical HFMD, the rashes were widely distributed and were diverse in morphology, which were in accordance with previously reported data in USA and Japan $[13,19,24]$. Thus, atypical HFMD is easy to misdiagnose as chickenpox, impetigo, and measles, which may delay its diagnosis and therapy. Rashes were also found on the face, trunk, and limbs besides palms, soles, and buttocks, $64.1 \%$ of children developed vesicles, and some children also had scabs in late phase. These manifestations were in agreement with HFMD caused by CV-A6 in Japan [24]. This suggests that clinicians should notice the atypical rashes, especially the vesicles-dominant rashes that cannot be explained by other diseases, because these might be manifestations of atypical HFMD, and pathogen detection of the stool is recommended.

In late phase, the incidences of onychomadesis and desquamation were relatively high. A lot of pathogens may cause onychomadesis and desquamation, but CV-A6 is a major pathogen, which was in accordance with findings reported in Taiwan [26]. Onychomadesis and desquamation occurred mainly at 4-5 weeks after the acute phase, which was in agreement with the time point reported by Österback et al. [9]. In addition, Österback et al. found CV-A6 was also detected in the shedding nails and thus they proposed that viral replication caused the destruction of nail matrix. However, Shikuma et al. [27] found onychomadesis occurred only in the fingers with severe skin lesions, which might be ascribed to the severe inflammation after skin injury. Whether onychomadesis is related to viral replication or inflammation or ascribed to the overprevention and overprotection (such as repeated hand-washing) [28] is still unclear, and further investigation is required.

In early studies on the etiology of HFMD, results showed EV-A71 and CV-A16 were the major pathogens $[3,4]$, and HFMD caused by EV-A71 and CV-A16 was prevalent alternatively in different regions. Since 2008, the incidence of HFMD caused by CV-A6 has been increasing in Southeast Asia and Europe [9-14]. In the present study, more than $65 \%$ of atypical HFMD was caused by CV-A6, which was consistent with that reported in Taiwan in 2010 [18]. These findings highlight the importance of CV-A6 in the pathogenesis of atypical HFMD. However, CV-A6 is not a common strain in Chongqing, and whether the CV-A6 increases over year in Chongqing is required to be further confirmed in future studies.

There were still limitations in the present study. First, not all the children with atypical HFMD were hospitalized for therapy, and thus the sample size of atypical children was still small. The clinical features collected from the hospitalized children with atypical HFMD might not represent the characteristics of all the children with atypical HFMD. In addition, some parents might neglect the onychomadesis and desquamation in late phase, which may underestimate the incidence of complications.

Taken together, the atypical HFMD has an obviously seasonal prevalence, special clinical manifestations, and a relatively good prognosis. Rashes in atypical HFMD are widely distributed and are diverse in morphology, the incidences of onychomadesis and desquamation are relatively high in late phase, and atypical HFMD is mainly caused by CV-A6 in Chongqing, China.

\section{Conflict of Interests}

The authors declare that there is no conflict of interests regarding the publication of this paper.

\section{Authors' Contribution}

Xiang Yan and Zhen-Zhen Zhang are equal contributors. 


\section{Acknowledgments}

The authors are grateful to Mei-Hua Li and Hui-Yin Mao in Department of Infection in Children's Hospital of Chongqing Medical University for providing and collecting samples and Fang-Fang Lai for providing excellent technical assistance.

\section{References}

[1] C. R. Robinson, F. W. Doane, and A. J. Rhodes, "Report of an outbreak of febrile illness with pharyngeal lesions and exanthem: Toronto, summer 1957-isolation of group A coxsackie virus," Canadian Medical Association Journal, vol. 79, no. 8, pp. 615-621, 1958.

[2] W. Xing, Q. Liao, C. Viboud et al., "Hand, foot, and mouth disease in China, 2008-12: an epidemiological study," The Lancet Infectious Diseases, vol. 14, no. 4, pp. 308-318, 2014.

[3] X. J. Tan and W. B. Xu, "Molecular epidemiological research on enterovirus 71," Chinese Journal of Vaccines and Immunization, vol. 4, pp. 361-367, 2008.

[4] S.-J. He, J.-F. Han, X.-X. Ding, Y.-D. Wang, and C.-F. Qin, "Characterization of enterovirus 71 and coxsackievirus A16 isolated in hand, foot, and mouth disease patients in Guangdong, 2010," International Journal of Infectious Diseases, vol. 17, no. 11, pp. e1025-e1030, 2013.

[5] Y.-Q. He, L. Chen, W.-B. Xu et al., "Emergence, circulation, and spatiotemporal phylogenetic analysis of coxsackievirus A6- and coxsackievirus A10-associated hand, foot, and mouth disease infections from 2008 to 2012 in Shenzhen, China," Journal of Clinical Microbiology, vol. 51, no. 11, pp. 3560-3566, 2013.

[6] J. Lu, H. Zeng, H. Zheng et al., "Hand, foot and mouth disease in Guangdong, China, in 2013: new trends in the continuing epidemic," Clinical Microbiology and Infection, vol. 20, no. 7, pp. O442-O445, 2014.

[7] Q.-B. Lu, X.-A. Zhang, Y. Wo et al., "Circulation of coxsackievirus A10 and A6 in hand-foot-mouth disease in China, 20092011," PLoS ONE, vol. 7, no. 12, Article ID e52073, 2012.

[8] G. Mandell, R. Dolin, and J. Bennett, Mandell, Douglas, and Bennett's Principles and Practice of Infectious Diseases, Elsevier, 2009.

[9] R. Österback, T. Vuorinen, M. Linna, P. Susi, T. Hyypiä, and M. Waris, "Coxsackievirus A6 and hand, foot, and mouth disease, Finland," Emerging Infectious Diseases, vol. 15, no. 9, pp. 14851488, 2009.

[10] M. A. Bracho, F. González-Candelas, A. Valero, J. Córdoba, and A. Salazar, "Enterovirus co-infections and onychomadesis after hand, foot, and mouth disease, Spain, 2008," Emerging Infectious Diseases, vol. 17, no. 12, pp. 2223-2231, 2011.

[11] T. Fujimoto, S. Iizuka, M. Enomoto et al., "Hand, foot, and mouth disease caused by coxsackievirus A6, Japan, 2011," Emerging Infectious Diseases, vol. 18, no. 2, pp. 337-339, 2012.

[12] J. Puenpa, T. Chieochansin, P. Linsuwanon et al., "Hand, foot, and mouth disease caused by coxsackievirus A6, Thailand, 2012," Emerging Infectious Diseases, vol. 19, no. 4, pp. 641-643, 2013.

[13] Centers for Disease Control and Prevention, "Notes from the field: severe hand, foot, and mouth disease associated with coxsackievirus A6-Alabama, Connecticut, California, and Nevada, November 2011-February 2012," Morbidity and Mortality Weekly Report, vol. 61, no. 12, pp. 213-214, 2012.
[14] Y. Wu, A. Yeo, M. C. Phoon et al., "The largest outbreak of hand; foot and mouth disease in Singapore in 2008: the role of enterovirus 71 and coxsackievirus A strains," International Journal of Infectious Diseases, vol. 14, no. 12, pp. e1076-e1081, 2010.

[15] Taiwan CDC website, November 2012, http://www.cdc.gov.tw/ professional/list.aspx?treeid=4C19A0252BBEF869\&nowtreeid= 34330F1263D5F7E4.

[16] J. Lu, H. Zeng, H. Zheng et al., "Hand, foot and mouth disease in Guangdong, China, in 2013. NEW trends in the continuing epidemic," Clinical Microbiology and Infection, vol. 20, no. 7, pp. O442-O445, 2014.

[17] J.-F. Han, S. Xu, Y. Zhang et al., "Hand, foot, and mouth disease outbreak caused by coxsackievirus A6, China, 2013," Journal of Infection, vol. 69, no. 3, pp. 303-305, 2014.

[18] W.-C. Huang, L.-M. Huang, C.-Y. Lu, A.-L. Cheng, and L.Y. Chang, "Atypical hand-foot-mouth disease in children: a hospital-based prospective cohort study," Virology Journal, vol. 10, article 209, 2013.

[19] E. F. Mathes, V. Oza, I. J. Frieden et al., “'Eczema coxsackium” and unusual cutaneous findings in an enterovirus outbreak," Pediatrics, vol. 132, no. 1, pp. e149-e157, 2013.

[20] S. X. Ge, Q. Yan, S. Z. He, S. J. Zhuang, J. Niu, and N. Xia, "Specific primer amplification of the VP1 region directed by 5 ' UTR sequence analysis: enterovirus testing and identification in clinical samples from hand-foot-and-mouth disease patients," Journal of Virological Methods, vol. 193, no. 2, pp. 463-469, 2013.

[21] Y. Huang, T. Deng, S. Yu et al., "Effect of meteorological variables on the incidence of hand, foot, and mouth disease in children: a time-series analysis in Guangzhou, China," BMC Infectious Diseases, vol. 13, no. 1, article 134, 2013.

[22] E. Ma, T. Lam, K. C. Chan, C. Wong, and S. K. Chuang, "Changing epidemiology of hand, foot, and mouth disease in Hong Kong, 2001-2009," Japanese Journal of Infectious Diseases, vol. 63, no. 6, pp. 422-426, 2010.

[23] L. W. Ang, B. K. Koh, K. P. Chan, L. T. Chua, L. James, and K. T. Goh, "Epidemiology and control of hand, foot and mouth disease in Singapore, 2001-2007," Annals of the Academy of Medicine Singapore, vol. 38, no. 2, pp. 106-112, 2009.

[24] M. Kobayashi, T. Makino, N. Hanaoka et al., "Clinical manifestations of coxsackievirus A6 infection associated with a major outbreak of hand, foot, and mouth disease in Japan," Japanese Journal of Infectious Diseases, vol. 66, no. 3, pp. 260-261, 2013.

[25] Y.-H. Kung, S.-W. Huang, P.-H. Kuo et al., "Introduction of a strong temperature-sensitive phenotype into enterovirus 71 by altering an amino acid of virus 3D polymerase," Virology, vol. 396, no. 1, pp. 1-9, 2010.

[26] S.-H. Wei, Y.-P. Huang, M.-C. Liu et al., "An outbreak of coxsackievirus A6 hand, foot, and mouth disease associated with onychomadesis in Taiwan, 2010," BMC Infectious Diseases, vol. 11, no. 1, article 346, 2011.

[27] E. Shikuma, Y. Endo, A. Fujisawa, M. Tanioka, and Y. Miyachi, "Onychomadesis developed only on the nails having cutaneous lesions of severe hand-foot-mouth disease," Case Reports in Dermatological Medicine, vol. 2011, Article ID 324193, 2 pages, 2011.

[28] E. Haneke, "Onychomadesis and hand, foot and mouth disease-is there a connection?" Euro Surveillance, vol. 15, no. 37, Article ID 19664, 2010. 


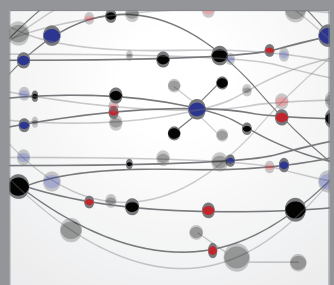

The Scientific World Journal
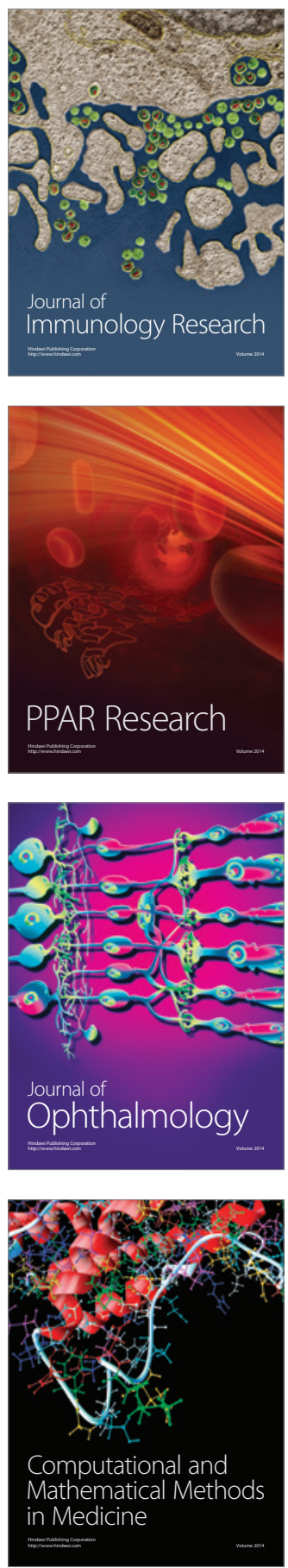

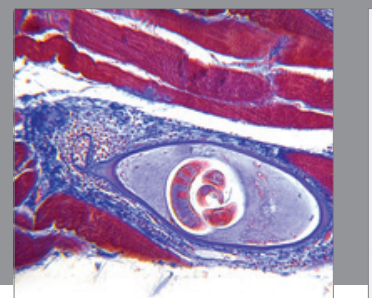

Gastroenterology

Research and Practice
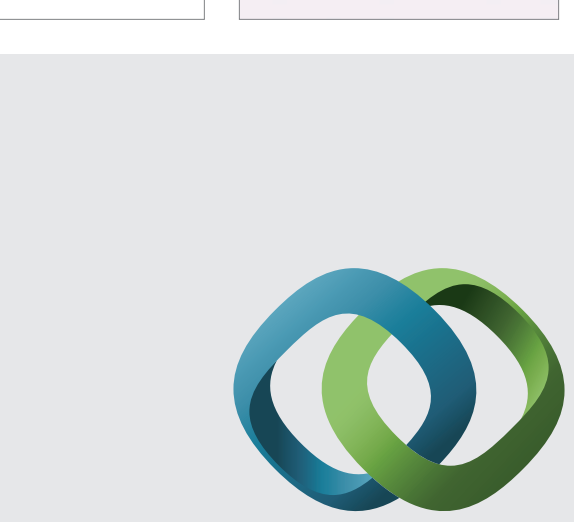

\section{Hindawi}

Submit your manuscripts at

http://www.hindawi.com
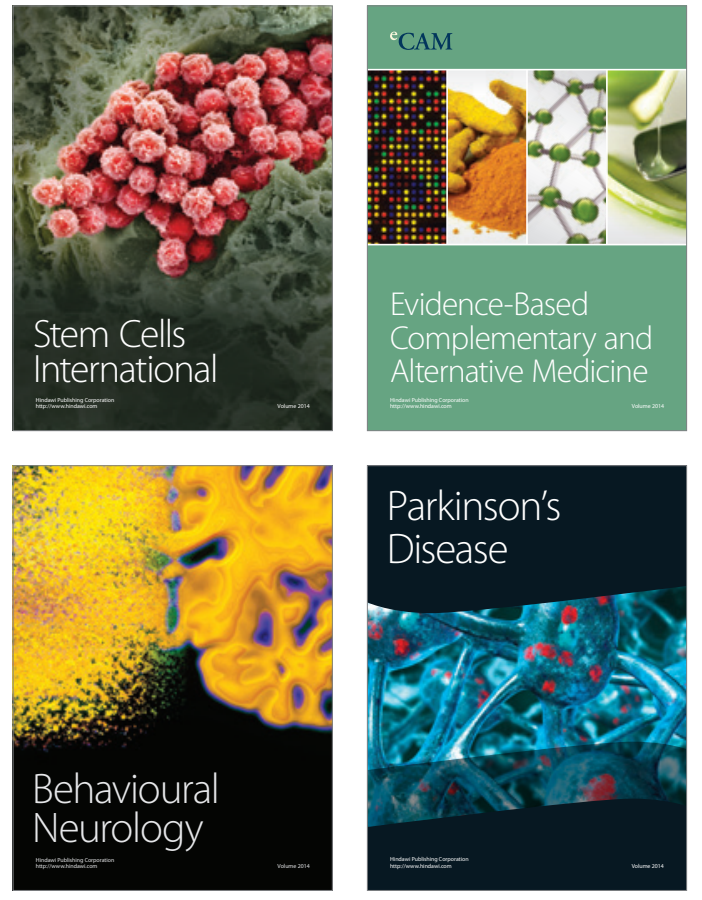
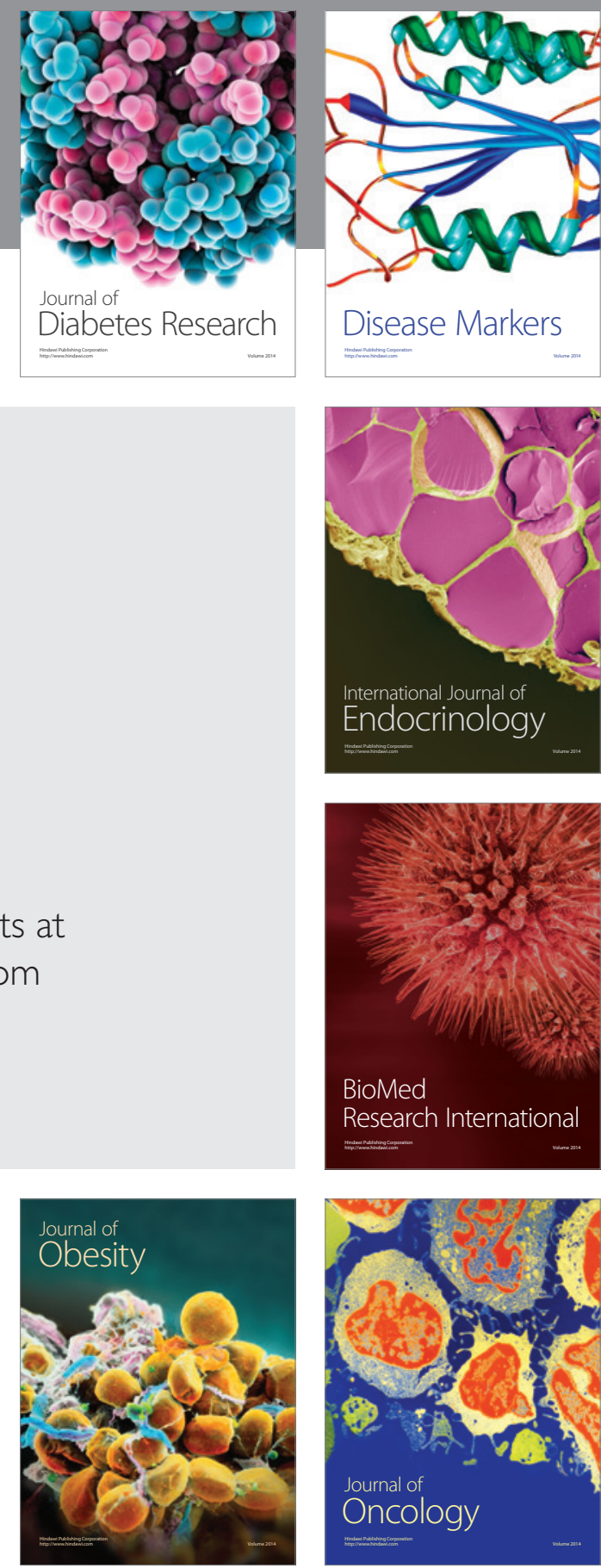

Disease Markers
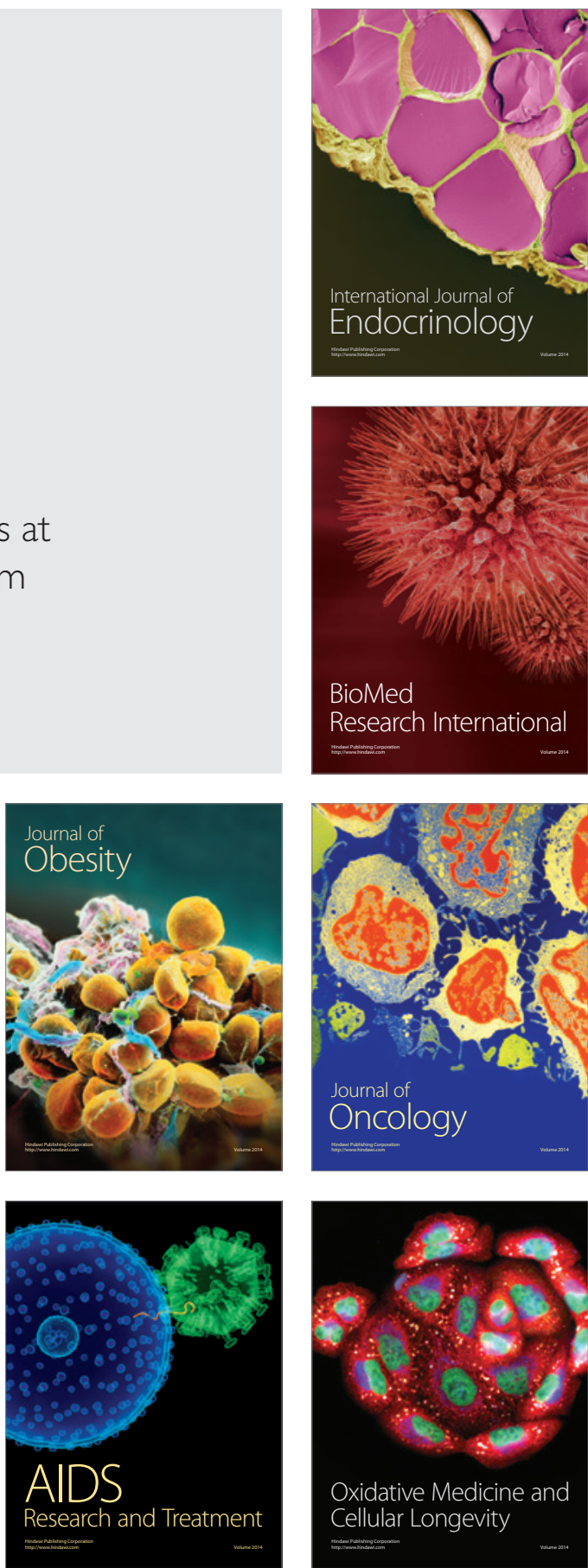